\title{
ON A CONJECTURE OF GRAHAM CONCERNING GREATEST COMMON DIVISORS
}

\author{
GERALD WEINSTEIN
}

ABSTRACT. Let $a_{1}<a_{2}<\cdots<a_{n}$ be a finite sequence of positive integers. R. L. Graham has conjectured that $\max _{i, j}\left\{a_{i} /\left(a_{i}, a_{j}\right)\right\} \geqslant n$. The following are proved:

(1) If $a_{l}=p$, a prime, for some $l$ and $p \neq\left(a_{i}+a_{j}\right) / 2,1 \leqslant i<j \leqslant n$, then the conjecture holds.

(2) Given a finite sequence of positive integers $k_{1}<k_{2}<\cdots<k_{m}$, where $k_{I}=p$, a prime, for some $I$ and $k_{m}<n$, consider the set of all positive integral multiples of $k_{1}, k_{2}, \ldots, k_{m}$ which are $<n$. Denote these multiples by $a_{1}<a_{2}<\cdots<a_{q}$. Define $P_{n}$ to be a set consisting of the integers $a_{1}<a_{2}<\cdots<a_{q}<a_{q+1}<\cdots<a_{q+r}$ where $r$ is maximal, such that $n \leqslant a_{q+1}$ and $\max _{i, j}\left\{a_{i} /\left(a_{i}, a_{j}\right)\right\}<n$. Thus

$$
P_{n}=P_{n}\left(k_{1}, k_{2}, \ldots, k_{m}\right) \text {. }
$$

Then

(a) $\left|P_{n}\right| \geqslant n$ for at most finitely many $n$.

(b) If $\left|P_{n}\right|<n$ for $n<$ l.c.m. $\left\{k_{1}, k_{2}, \ldots, k_{m}\right\}$ then $\left|P_{n}\right|<n$ for all positive integers $n$.

In this note we consider the following conjecture of R. L. Graham [1]. Let $a_{1}<a_{2}<\cdots<a_{n}$ be a finite sequence of positive integers. Then $\max _{i, j}\left\{a_{i} /\left(a_{i}, a_{j}\right)\right\} \geqslant n$.

This conjecture has attracted many investigators and has been verified in the following special cases:

(a) $a_{i}$ is square-free for all $i$ (Marica and Schönheim [2]).

(b) $a_{1}$ is prime (Winterle [3]).

(c) $n$ is prime (Szemerédi [4]).

(d) $n-1$ is prime (Vélez [4]).

Vélez [4] also considers another conjecture of Graham as to the form of those sequences for which equality is achieved and shows that this second conjecture implies the title conjecture.

Here we prove two generalizations of Winterle's result (b).

Part I.

THEOREM 1. If $A$ is the sequence $a_{1}<a_{2}<\cdots<a_{n}$, where $a_{k}=p$, $a$ prime, for some $k$ and $p \neq\left(a_{i}+a_{j}\right) / 2,1 \leqslant i<j \leqslant n$, then

$$
\max _{i, j}\left\{a_{i} /\left(a_{i}, a_{j}\right)\right\} \geqslant n \text {. }
$$

Received by the editors January 19, 1976.

AMS (MOS) subject classifications (1970). Primary 10A05. 
Proof. We assume that $\max _{i j}\left\{a_{i} /\left(a_{i}, a_{j}\right)\right\}<n$ so that

$$
a_{n} \leqslant(n-1)\left(a_{n}, p\right) \leqslant(n-1) p .
$$

Let $B$ be the subsequence $b_{1}<b_{2}<\cdots<b_{r}$ consisting of all terms in $A$ not divisible by $p$. Since we may assume that the g.c.d. of the terms of $A$ is 1 , $B$ is not empty and $b_{r} \leqslant n-1$. We define a mapping $T$ by $T\left(b_{i}\right)=p^{h}\left|b_{i}-p\right|$, where $h$ is the largest nonnegative integer such that $P^{h}\left|b_{i}-p\right| \leqslant(n-1) p$. Since $\left|b_{i}-p\right|<n-1, h$ is always positive, hence $p \mid T\left(b_{i}\right)$ for all $b_{i}$. Since $\left(p, b_{i}\right)=1$ and $\left(\left|b_{i}-p\right|, b_{i}\right)=1$, we have $\left(b_{i}\right.$, $\left.T\left(b_{i}\right)\right)=1$ for all $b_{i}$. Also, if $T\left(b_{i}\right) \leqslant n-1$, then $p T\left(b_{i}\right) \leqslant(n-1) p$, which contradicts the definition of $T\left(b_{i}\right)$. Thus $T\left(b_{i}\right) \geqslant n$ for all $b_{i}$. These two results imply that $T\left(b_{i}\right)$ is never a term of the sequence $A$.

Now assume that $T\left(b_{i}\right)=T\left(b_{j}\right)$. Then $p^{h}\left|b_{i}-p\right|=p^{k}\left|b_{j}-p\right|$ for some $h$ and $k$. Since $p$ does not divide either $\left|b_{i}-p\right|$ or $\left|b_{j}-p\right|$, we see that $h=k$ and so $b_{i}-p= \pm\left(b_{j}-p\right)$. Since the minus sign contradicts the hypothesis, we must have $b_{i}=b_{j}$.

We now define $F\left(a_{i}\right)$, for each $a_{i} \in A$, as follows:

$$
\begin{array}{ll}
F\left(a_{i}\right)=a_{i} & \text { if } p \mid a_{i}, \\
F\left(a_{i}\right)=T\left(a_{i}\right) & \text { if } a_{i} \in B .
\end{array}
$$

Then $p \mid F\left(a_{i}\right) \leqslant(n-1) p$ for all $a_{i} \in A$. Also, since $T$ is $1-1$ and never maps $b_{i}$ into $A-B$, it follows that $F$ is $1-1$. Hence $|A| \leqslant n-1$; this contradicts the fact that $|A|=n$ and so completes the proof.

Corollary 1 (WINTERLE). Given the sequence $a_{1}<a_{2}<\cdots<a_{n}$, where $a_{1}=p$, a prime, then $\max _{i, j}\left\{a_{i} /\left(a_{i}, a_{j}\right)\right\} \geqslant n$.

Corollary 2. Given the sequence $a_{1}<a_{2}<\cdots<a_{n}$, where $a_{k}=p$ and $a_{k+1} \geqslant 2 p$, for some $k, p$ a prime, then $\max _{i, j}\left\{a_{i} /\left(a_{i}, a_{j}\right)\right\} \geqslant n$.

Proof. In both corollaries it is clear that $p \neq\left(a_{i}+a_{j}\right) / 2$ for $1 \leqslant i<j \leqslant$ $n$.

Part II. If we omit the condition of Theorem 1 that $p \neq\left(a_{i}+a_{j}\right) / 2$, $1 \leqslant i<j \leqslant n$, we can still show that the conjecture is "ultimately" true in the following sense.

Given a finite sequence of positive integers $k_{1}<k_{2}<\cdots<k_{m}$ where $k_{l}=p$, a prime, for some $I$ and $k_{m}<n$, consider the set of all positive integral multiples of $k_{1}, k_{2}, \ldots, k_{m}$ which are $<n$. Denote these multiples by $a_{1}<a_{2}<\cdots<a_{q}$. Define $P_{n}$ to be a set consisting of the integers $a_{1}<a_{2}<\cdots<a_{q}<a_{q+1}<\cdots<a_{q+r}$, where $r$ is maximal such that $n \leqslant a_{q+1}$ and $\max _{i, j}\left\{a_{i} /\left(a_{i}, a_{j}\right)\right\}<n$. Thus $P_{n}=P_{n}\left(k_{1}, k_{2}, \ldots, k_{m}\right)$. Then we have

THEOREM 2. (a) $\left|P_{n}\right| \geqslant n$ for at most finitely many $n$.

(b) If $\left|P_{n}\right|<n$ for $n<$ l.c.m. $\left\{k_{1}, k_{2}, \ldots, k_{m}\right\}$ then $\left|P_{n}\right|<n$ for all positive integers $n$. 
Several remarks are necessary before proceeding to the proof.

(1) We use the following notation:

$\left[k_{i}, k_{j}\right]$ : the least common multiple of $k_{i}$ and $k_{j}$;

$\phi(n)$ : the number of positive integers less than, and relatively prime to, $n$.

(2) We say that $k_{1}<k_{2}<\cdots<k_{m}$ is a basis which generates $P_{n}$.

(3) Define $I_{n}^{j}=\left\{x \in P_{n},(j-1) n \leqslant x<j n\right\}, j=1,2, \ldots, a_{1}\left(a_{1}=k_{1}\right)$.

Thus $P_{n}=\bigcup_{j=1}^{a_{1}} I_{n}^{j}$.

(4) There is no loss in generality if we choose $\left(k_{j}, p\right)=1$ for $k_{j} \neq p$.

(5) The number of elements in $I_{n}^{1}$ is given by

$$
\begin{aligned}
\left|I_{n}^{1}\right|= & {\left[\frac{n}{k_{1}}\right]+\cdots+\left[\frac{n}{k_{m}}\right]-\left(\left[\frac{n}{\left[k_{1}, k_{2}\right]}\right]+\cdots+\left[\frac{n}{\left[k_{i}, k_{j}\right]}\right]+\ldots\right) } \\
& +\left[\frac{n}{\left[k_{i}, k_{j}, k_{l}\right]}\right]+\cdots+(-1)^{m+1}\left[\frac{n}{\left[k_{1}, \ldots, k_{m}\right]}\right] .
\end{aligned}
$$

Define

$$
\begin{aligned}
d\left(I_{n}^{1}\right)= & \frac{1}{k_{1}}+\cdots+\frac{1}{k_{m}}-\left(\frac{1}{\left[k_{1}, k_{2}\right]}+\cdots+\frac{1}{\left[k_{i}, k_{j}\right]}+\ldots\right) \\
& +\frac{1}{\left[k_{i}, k_{j}, k_{l}\right]}+\cdots+\frac{(-1)^{m+1}}{\left[k_{1}, \ldots, k_{m}\right]},
\end{aligned}
$$

and

$$
\begin{aligned}
d\left(I_{n}^{2}\right)= & 1-\left(\frac{\phi\left(k_{1}\right)}{k_{1}}+\cdots+\frac{\phi\left(k_{m}\right)}{k_{m}}\right) \\
& +\left(\frac{\phi\left(\left[k_{1}, k_{2}\right]\right)}{\left[k_{1}, k_{2}\right]}+\cdots+\frac{\phi\left(\left[k_{i}, k_{j}\right]\right)}{\left[k_{i}, k_{j}\right]}+\ldots\right) \\
& -\left(\ldots+\frac{\phi\left(\left[k_{i}, k_{j}, k_{l}\right]\right)}{\left[k_{i}, k_{j}, k_{l}\right]}+\ldots\right)+(-1)^{m} \frac{\phi\left(\left[k_{1}, \ldots, k_{m}\right]\right)}{\left[k_{1}, \ldots, k_{m}\right]} .
\end{aligned}
$$

Then,

$$
\begin{aligned}
\frac{1}{n}\left|I_{n}^{1}\right| \leqslant \frac{1}{n}\left\{\frac{n}{k_{1}}+\cdots+\frac{n}{k_{m}}-\left(\frac{n}{\left[k_{1}, k_{2}\right]}+\cdots+\frac{n}{\left[k_{i}, k_{j}\right]}+\ldots\right)\right. \\
\left.\quad+\frac{n}{\left[k_{i}, k_{j}, k_{l}\right]}+\cdots+(-1)^{m+1} \frac{n}{\left[k_{1}, \ldots, k_{m}\right]}+2^{m-1}\right\} \\
=d\left(I_{n}^{1}\right)+\frac{2^{m-1}}{n} .
\end{aligned}
$$

Also, 


$$
\begin{aligned}
& \frac{1}{n}\left|I_{n}^{2}\right| \leqslant \frac{1}{n}\{n-\left(\frac{n}{k_{1}} \phi\left(k_{1}\right)+\cdots+\frac{n}{k_{m}} \phi\left(k_{m}\right)\right) \\
&+\left(\frac{n}{\left[k_{1}, k_{2}\right]} \phi\left(\left[k_{1}, k_{2}\right]\right)+\cdots+\frac{n}{\left[k_{i}, k_{j}\right]} \phi\left(\left[k_{i}, k_{j}\right]\right)+\ldots\right) \\
&+\cdots+(-1)^{m} \frac{n}{\left[k_{1}, \ldots, k_{m}\right]} \phi\left(\left[k_{1}, \ldots, k_{m}\right]\right) \\
&+\sum_{i=1}^{m} \phi\left(k_{i}\right)+\sum_{i, j}^{m} \phi\left(\left[k_{i}, k_{j}\right]\right) \\
&\left.+\sum_{i, j, l}^{m} \phi\left(\left[k_{i}, k_{j}, k_{l}\right]\right)+\cdots+\phi\left(\left[k_{1}, \ldots, k_{m}\right]\right)\right\} \\
& \leqslant d\left(I_{n}^{2}\right)+\left(k_{1} k_{2} \ldots k_{m}\right) / n .
\end{aligned}
$$

Note. This implies $n^{-1}\left|I_{n}^{j}\right| \leqslant d\left(I_{n}^{2}\right)+\left(k_{1} k_{2} \cdots k_{m}\right) / n, 2 \leqslant j \leqslant k_{1}$.

We need four lemmas.

Lemma 1. Let $2<b_{1}<b_{2}<\cdots<b_{r}$ be integers. Then

$$
\begin{aligned}
1 / b_{1} & +1 / b_{2}+\cdots+1 / b_{r}-\left(1 /\left[b_{1}, b_{2}\right]+\cdots+1 /\left[b_{i}, b_{j}\right]+\ldots\right) \\
& +\cdots+1 /\left[b_{i}, b_{j}, b_{k}\right]+\cdots+(-1)^{r+1} /\left[b_{1}, \ldots, b_{r}\right] \\
< & \phi\left(b_{1}\right) / b_{1}+\phi\left(b_{2}\right) / b_{2}+\cdots+\phi\left(b_{r}\right) / b_{r} \\
& -\left(\phi\left(\left[b_{1}, b_{2}\right]\right) /\left[b_{1}, b_{2}\right]+\cdots+\phi\left(\left[b_{i}, b_{j}\right]\right) /\left[b_{i}, b_{j}\right]+\cdots\right)+\cdots \\
& +\phi\left(\left[b_{i}, b_{j}, b_{k}\right]\right) /\left[b_{i}, b_{j}, b_{k}\right]+\cdots \\
& +(-1)^{r+1} \Phi\left(\left[b_{1}, \ldots, b_{r}\right]\right) /\left[b_{1}, \ldots, b_{r}\right] .
\end{aligned}
$$

Proof. Define $L=\left[b_{1}, b_{2}, \ldots, b_{r}\right]$. Let $M$ be the set of all multiples of $b_{1}$, $b_{2}, \ldots, b_{r}$ which are less than or equal to $L$. Let $N$ be the set of all numbers which are relatively prime to at least one of $b_{1}, b_{2}, \ldots, b_{r}$ and less than or equal to $L$. Then the inequality is equivalent to the statement $|M|<|N|$. To see that the latter is true, define

$$
A_{i}=\bigcup_{k=0}^{\left(L / b_{i}\right)-1}\left(b_{i}-1\right)+k b_{i} \text { and } S=\bigcup_{i=1}^{r} A_{i} \text {. }
$$

Then $S \subset N$ and $|S|=|M|$. But $1 \in(N-S)$ implies $|S|<|N|$ and so $|M|<|N|$.

LEMMA 2. Let $k$ and $p$ be integers, $A$ and $B$ real numbers, such that $1 \leqslant k<p$ and $0<A<B<1$. Then $A+(1-A) / p+k(1-B) / p<1$. 
Proof. Equivalently, we must show $p^{-1}((p-1) A+1+k-k B)<1$. If we define $\varepsilon=B-A>0$ this becomes $(p-k-1) A+k(1-\varepsilon)+1<p$. But

$$
\begin{aligned}
(p-k-1) A+k(1-\varepsilon)+1 & \leqslant p-k-1+k-\varepsilon k+1 \\
& =p-\varepsilon k<p .
\end{aligned}
$$

LEMMA 3. Let $A$ be a basis consisting of the positive integers $k_{1}<\cdots<k_{m}$ and let $B=A \cup\{p\}$ be another basis where $p$ is a prime which does not divide any $k_{j}$. Let $d\left(I_{n}^{1}\right)_{A}$ denote this previously defined function on the basis $A$. Similarly we define $d\left(I_{n}^{1}\right)_{B}, d\left(I_{n}^{2}\right)_{A}$, and $d\left(I_{n}^{2}\right)_{B}$. Then

$$
\begin{gathered}
d\left(I_{n}^{1}\right)_{B}=d\left(I_{n}^{1}\right)_{A}+p^{-1}\left(1-d\left(I_{n}^{1}\right)_{A}\right), \\
d\left(I_{n}^{2}\right)_{B}=d\left(I_{n}^{2}\right)_{A}-(\phi(p) / p) d\left(I_{n}^{2}\right)=p^{-1} d\left(I_{n}^{2}\right)_{A} .
\end{gathered}
$$

Proof. (1) Regroup terms of $d\left(I_{n}^{1}\right)_{B}$, taking first those without $p$, then those with $p$. Since $p$ is relatively prime to all $k_{j}, 1 / p$ may be factored from each term in the second group to leave a term identical to one in the first group but with opposite signs except for the first term.

(2) Regroup as above. This time $\phi(p) / p$ may be factored from each term in the second group.

Lemma 4. Consider again the basis $B$ defined in Lemma 3 , let $L=\left[k_{1}\right.$, $\left.k_{2}, \ldots, p, \ldots, k_{m}\right]$ and let $P_{n}$ be the set generated by $B$. Then $\left|P_{n}\right|$ is a linear function on the set $r+k L, k=0,1,2, \ldots$, for any integer $r \geqslant 0$.

Proof. Define

$$
\begin{aligned}
X\left(n ; a_{1}, a_{2}, \ldots, a_{N}\right)=\left[n / a_{1}\right]+\cdots+\left[n / a_{N}\right] \\
-\left(\left[n /\left[a_{1}, a_{2}\right]\right]+\cdots+\left[n /\left[a_{i}, a_{j}\right]\right]+\ldots\right)+\ldots \\
+\left[n /\left[a_{i}, a_{j}, a_{l}\right]\right]+\ldots+(-1)^{N+1}\left[n /\left[a_{1}, \ldots, a_{N}\right]\right]
\end{aligned}
$$

Then

$$
\begin{gathered}
\left|I_{n}^{1}\right|=X\left(n ; k_{1}, \ldots, p, \ldots, k_{m}\right), \\
\left|I_{n}^{j}\right|=X\left(j n ; s_{1}^{(j)}, \ldots, s_{N_{j}}^{(j)}\right)-X\left((j-1) n ; s_{1}^{(j)}, \ldots, s_{N_{j}}^{(j)}\right), \quad \text { if } 2 \leqslant j \leqslant k_{1},
\end{gathered}
$$

where $s_{l}^{(j)} \mid L$ and $\left(s_{l}^{(j)}, k_{i}\right) \geqslant j$ for every $i$. Thus

$$
\begin{aligned}
\left|P_{n}\right|= & \sum_{j=1}^{k_{1}}\left|I_{n}^{j}\right|=X\left(n ; k_{1}, \ldots, p, \ldots, k_{m}\right) \\
& +\sum_{j=2}^{k_{1}} X\left(j n ; s_{1}^{(j)}, \ldots, s_{N_{j}}^{(j)}\right)-X\left((j-1) n ; s_{1}^{(j)}, \ldots, s_{N_{j}}^{(j)}\right) .
\end{aligned}
$$

Since every term in this sum is of the form $[j n / a]$, where $a \mid L$, and

$$
[j(r+k L) / a]=[j r / a]+k[j L / a]
$$

we have 


$$
\left|P_{r+k L}\right|=\left|P_{r}\right|+k\left|P_{L}\right|
$$

Now we may proceed directly to the

Proof of Theorem 2. Let $B$ denote the basis $k_{1}<\cdots<k_{i}=p$ $<\cdots<k_{m}$ and let $A$ denote the basis $k_{1}<\cdots<k_{i-1}<k_{i+1}<\cdots<$ $k_{m}$. Remark (4) permits us to take $p$ relatively prime to all other $k_{j}$ without loss of generality. We wish to find an upper bound for $\left|P_{n}\right|$ when the basis is $B$.

$$
\begin{aligned}
n^{-1}\left|P_{n}\right|= & \frac{1}{n} \sum_{j=1}^{k_{1}}\left|I_{n}^{j}\right| \\
\leqslant & \left\{d\left(I_{n}^{1}\right)_{B}+\frac{2^{m-1}}{n}+\left(k_{1}-1\right)\left(d\left(I_{n}^{2}\right)_{B}+\frac{k_{1} k_{2} \cdots k_{m}}{n}\right)\right\} \\
= & \left\{d\left(I_{n}^{1}\right)_{A}+p^{-1}\left(1-d\left(I_{n}^{1}\right)_{A}\right)+\left(k_{1}-1\right)\left(p^{-1}\right) d\left(I_{n}^{2}\right)_{A}\right\} \\
& +\left(2^{m-1}+\left(k_{1}-1\right) k_{1} \cdots k_{m}\right) / n, \text { by Lemma 3. }
\end{aligned}
$$

By Lemma $1, d\left(I_{n}^{1}\right)_{A}<1-d\left(I_{n}^{2}\right)_{A}$, and so Lemma 2 implies the last \{\}$<1$. Hence if $n$ is sufficiently large we have $n^{-1}\left|P_{n}\right|<1$, which proves the first part of the theorem.

Let $L$ denote the 1.c.m. of the basis $B$ and define $f(n)=\left|P_{n}\right|-n$. By Lemma $4, f(n)$ is a linear function for $n=k L, k=0,1,2, \ldots$, and, from what we have just shown, $f(n)$ is a nonincreasing linear function on these values of $n$. Hence $f(L)<0$. But $f(n+k L)=f(n)+k f(L)$, so that $f(n)<$ 0 implies $f(n+k L)<0$. This proves the second part of the theorem.

\section{REFERENCES}

1. R. L. Graham, Unsolved problem 5749, Amer. Math. Monthly 77 (1970), 775.

P. Erdö, Problems and results on combinatorial number theory, A Survey of Combinatorial Theory, Chap. 12, North-Holland, Amsterdam, 1973. MR 50 \# 12957.

2. J. Marica and J. Schönheim, Differences of sets and a problem of Graham, Canad. Math. Bull. 12 (1969), 635-637. MR 40 \#2633.

3. Riko Winterle, A problem of $R$. L. Graham in combinatorial number theory, Proc. Louisiana Conf. on Combinatorics, Graph Theory and Computing (Louisiana State Univ., Baton Rouge, La., 1970), Louisiana State Univ., Baton Rouge, La., 1970, pp. 357-361. MR 42 \#3051.

4. W. Y. Vélez, Some remarks on a number theoretic problem of Graham, Acta Arith. (to appear).

Department of Mathematics, City College, New York, New York 10031 